\title{
A MOVABLE JAW MODEL FOR THE HUMAN FACE*
}

\author{
UG̃UR GÜDÜKBAY \\ Department of Computer Engineering and Information Science, Bilkent University, 06533 Bilkent. \\ Ankara, Turkey \\ e-mail: gudukbay(a)cs.bilkent.edu.tr
}

\begin{abstract}
Although there is a great deal of work on facial animation, there is not much research on the effect of jaw motion on the movement of the face. The complex nature of the jaw bones makes it difficult to implement all the motions the jaw can do. The human jaw has two widely separated identical joints behaving like a single joint. Widely separated joints of the mandible (lower jaw bone) allow it to translate in any direction and/or rotate about any axis in three-dimensional space although its movements are somewhat restricted by physical constraints and patterns of muscle aztivity. A simplified jaw model which covers the major movements of the jaw is proposed in this paper. The lower jaw in the model can rotate around the axis connecting the two ends of the jaw and make small translational motions in any direction in 3-D space. The face is modeled as a two layer model which is attached to the jaw. The inner layer of the face moves kinematically as dictated by the jaw. The outer layer moves with the effect of the springs connecting it to the inner layer. The motion of the outer layer is calsulated using spring-mass equations. Eating and chewing actions are simulated as applications of the model. 1997 Elsevier Science Lid. All rights reserved
\end{abstract}

\section{INTRODUCTION}

A movable jaw model for the human face for simulating eating and chewing actions is developed in this paper. The model is implemented in $J_{a c k}{ }^{\dagger}[1]$, which is a system for displaying and manipulating articulated geometric objects. The main problem for chewing is to do appropriate jaw motions while the mouth stays closed and the face skin remains connected and realistic. The face model is composed of upper and lower jaw bones, upper and lower teeth attached to the jaw bones, and a two-layer skin model. The inner layer of the face is attached to the jaw bones and moves kinematically as dictated by the jaw bones. The outer layer is attached to the inner layer with springs. Each outer layer node is also attached to its neighbors with springs.

The human lower jaw articulates with the upper jaw by two widely separated joints, which could be described as the two halves of a single joint. The movements of the mandible are complex because to a limited extent it can translate in any direction and/or rotate about any axis in 3-D space. This structure, together with the associated jaw muscles, allow human (and other mammals) to process food very effectively [2].

The jaw is modeled as a joint which can rotate around an axis connecting the two ends of the jaw bone and make small translational motions in any direction in 3-D space as in the real jaw. In this way, it

\footnotetext{
*Work done while the author was with the Department of Computer and Information Science, University of Pennsylvania. Philadelphia, PA, U.S.A.

${ }^{+} J_{a c k} k^{B}$ is a registered trademark of the University of Pennsylvania
}

behaves as two widely separated joints, each can rotate around the axis connecting them, and the lower jaw bone can make small translational motions.

The inner ayer of the face is modeled as two pieces; the upper part and lower part. The face is separated into two parts along the mouth. When this layer moves by the movement of the jaw bones, the outer layer also moves as dictated by the springs between outer and inner layer nodes. The springs connecting the outer layer nodes to their neighbors provides that the outer face layer (face skin) remains smooth and connected during jaw motion. A simplified view of the two-layer face model is shown in Fig. 1.

\section{PREVIOUS WORK}

Previous efforts for facial animation [3] can be divided intc three broad categories: parametric models [4]; structure-based models [5]; and physicsbased models [6]. In parametric- and structure-based models, the effect of jaw motion on facial animation is not addressed. Terzopoulos and Waters developed a physically-based hierarchical model of the human face [6]. The model incorporates a physically-based approximation to facial tissue and a set of anatomically-motivated facial muscle actuators. The synthetic tissue is a layered deformable lattice of point masses connected by elastic springs. A numerical simulation computes large-scale tissue deformation by continuously propagating through the lattice the local stresses induced by activated muscle fibers. The two-layer spring-mass model described here is similar to Terzopoulos and Waters' model except that it has a movable jaw whose parameter motion is kinematic. The motion of the face skin is determined by the spring forces due to the jaw motion instead of 


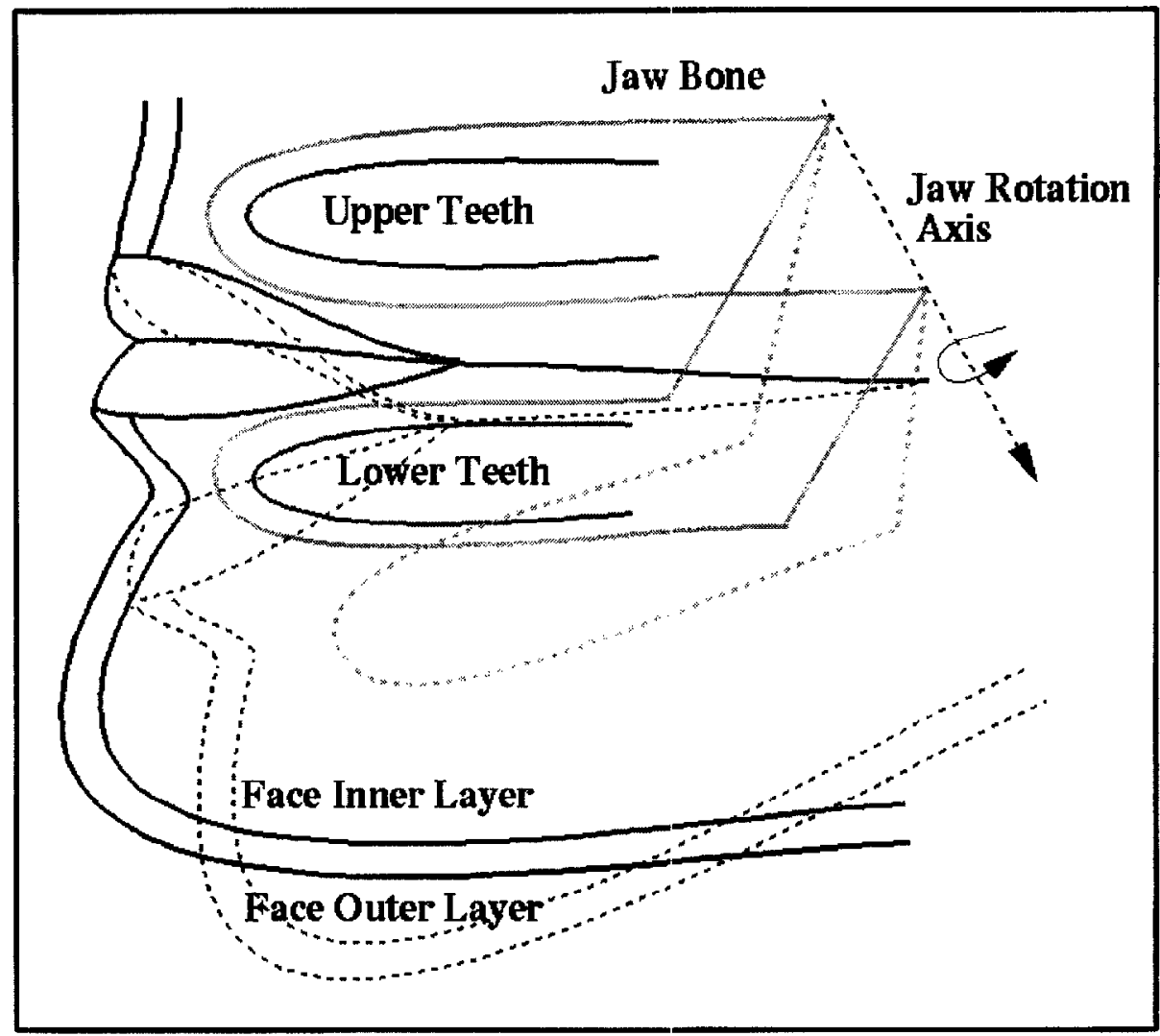

\section{Initial Position}

\section{Final Position}

Fig. 1. A simplified view of the two-layer face model.

anatomically-motivated facial muscle actuators. Although Baragar and Osborn [2] have described a jaw model which relates patterns of human jaw movement to biomechanical constraints, nobody has incorporated a truly dynamic model of the jaw into facial models to the best of the author's knowledge. This is because of the difficulty of the task. Using a jaw model whose motion is kinematic is easier to incorporate into face models.

Kunii et al. [7] propose approaches for an automated evaluation of jaw occlusion and chewing ability. Their approaches are for the evaluation of the load on teeth and of the grinding process and can be used for computer-aided diagnosis of occlusal disorders. However, they address the problem of jaw motion modeling as a future research subject. I propose a mechanism for motions of the lower jaw which involves plain interpolation of positions of the lower jaw as a rigid body as stated in their paper as a future work.

\section{FACE SKIN GENERATION DURING JAW MOTION}

When the lower part of the inner layer of the face model moves as a rigid body with jaw rotation, a gap occurs between the upper and lower part of the inner layer. This gap is closed by the outer layer which is like real face skin (see Fig. 1).
To generate face skin points during the jaw motion, a spring-mass system on skin points is formed. When the jaw moves kinematically for each time step of an animation, the inner layer of the face also moves as dictated by the jaw. The outer layer points, which are attached to the inner layer points, move with the effect of springs connecting them to their neighbors and inner layer points, until the springs come to rest. In other words, when the jaw moves a little, the differential equation solver iterates until the springs come to rest (when the kinetic energy of the nodes is below a certain threshold value).

In order to get a stable spring-mass system. each outer layer point is connected to all of its neighbors using springs as well as to the inner layer 'anchoring points'. To make the outer layer points resistant to twisting and shearing, each point is also connected to the anchoring points of its neighbors. The spring connections between the skin points and their neighbors and between the skin points and corresponding inner layer points are shown in Fig. 2.

\subsection{Spring-mass equations}

The equations of motion for the face skin with $n$ nodes can be written in Lagrange's form as follows: 


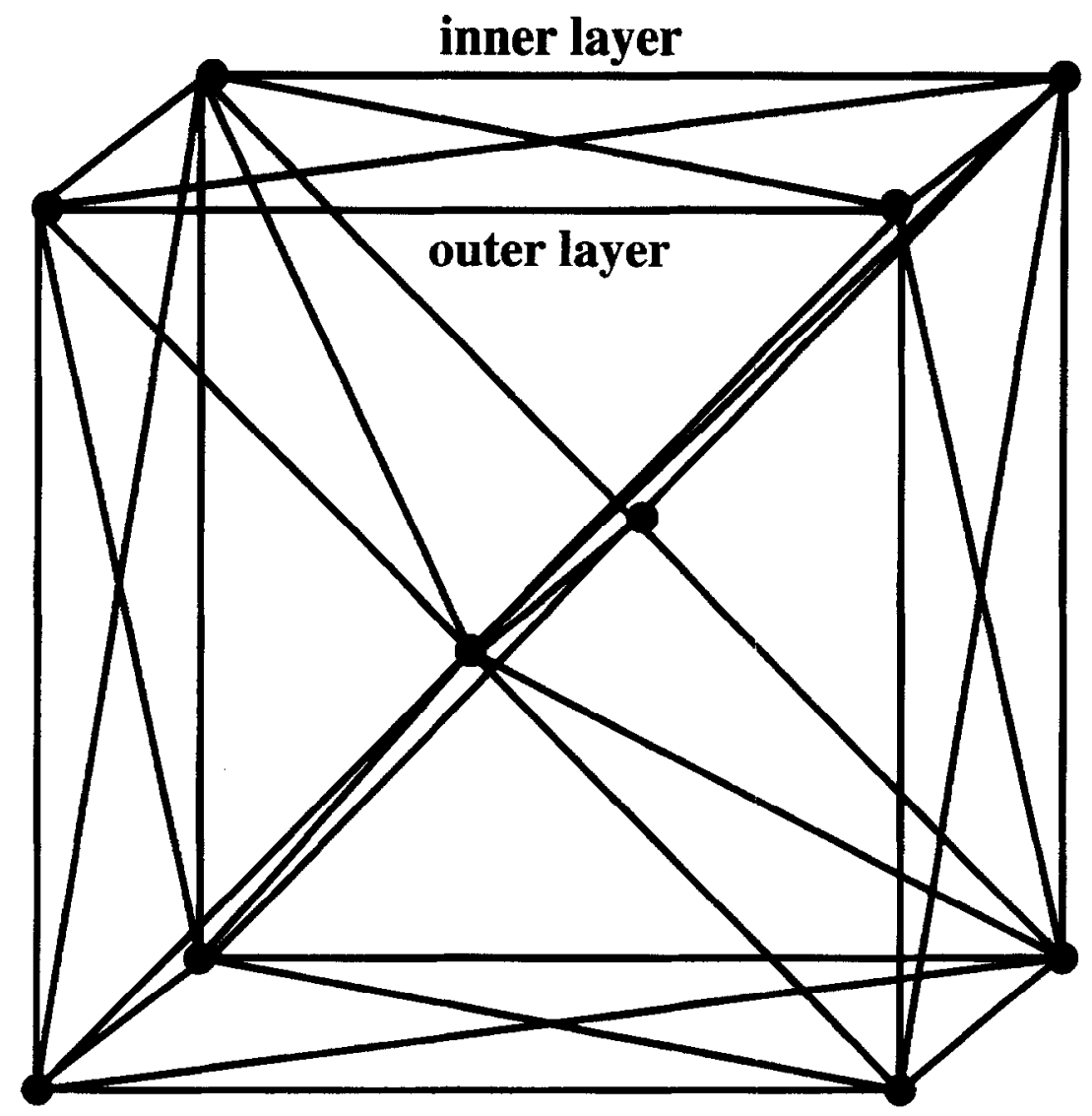

Fig. 2. The spring connections between a skin point and its ne ghbors and inner layer points

$$
\mathbf{M} \frac{d^{2}}{d t^{2}} \mathbf{x}+\mathbf{C} \frac{d}{d t} \mathbf{x}+\mathbf{f}_{K}(\mathbf{x})=\mathbf{f}(\mathbf{x})
$$

where $\mathbf{x}$ is the position vector for the model points ( $T$ denotes the transpose):

$$
\mathbf{x}^{T}=\left[\mathbf{x}_{1}^{T} \mathbf{x}_{2}^{T} \mathbf{x}_{n}^{T}\right]
$$

and $\mathbf{x}_{i}$ represents the position vector of the point $i$, where $i=1,2 \ldots, n$.

$\mathbf{M}$ is the mass matrix, an $n \times n$ diagonal matrix which contains masses of the model points as diagonal elements, and $\mathbf{C}$ is the damping matrix, an $n \times n$ diagonal matrix which contains velocity-dependent damping coefficients of the model points as diagonal elements. Velocity-dependent damping elements dissipate the kinetic energy in the model so that the model comes to rest after some number of iterations. The velocity-dependent damping coefficients are set in such a way that the face model behaves in a critically damped manner to achieve fast convergence.

$f(x)$ is the external force vector and $\mathbf{f}_{K}$ is the vector of spring forces acting on the model points to represent elastic properties of the model. Since I move the lower jaw kinematically, the external forces acting on the skin points are zero. In other words, the inner layer of the face moves with the lower jaw, which is attached to it kinematically, and the outer layer of the face moves dynamically as dictated by the spring forces connecting its points to the inner layer.

The spring forces between a pair of particles at positions a and b (Hooke's law spring) are [8]

$$
\mathbf{f}_{a}=-k_{s} \frac{(\| \mathbf{I} \mid-r)}{\|\mathbf{l}\|} \mathbf{l}, \mathbf{f}_{b}=-\mathbf{f}_{a}
$$

where $\mathbf{f}_{a}$ and $\mathbf{f}_{b}$ are the forces on $\mathbf{a}$ and $\mathbf{b}$. respectively, $\|=\mathbf{a}-\mathbf{b}, r$ is the rest length, and $k_{s}$ is a spring constant. In Equation (3), the spring force magnitude is proportional to the difference between the actual length and the rest length.

The total spring force acting on a point $i$ in the model is the sum of the individual spring forces between the point and its neighbors and also between the point and its anchor points on the inner layer of the face model $\left(f_{i j}\right.$ is the spring force exerted by node $j$ on node $i$ ):

$$
\mathbf{f}_{K}(i)=\sum_{i \in N_{i}} \mathbf{f}_{i j}+\sum_{k \in A_{i}} \mathbf{f}_{i k}
$$

where $N_{i}$ are neighbors of node $i$ and $A_{i}$ are anchors of node $i$ on the inner layer of the face.

The dynamics of the deformable face model are 


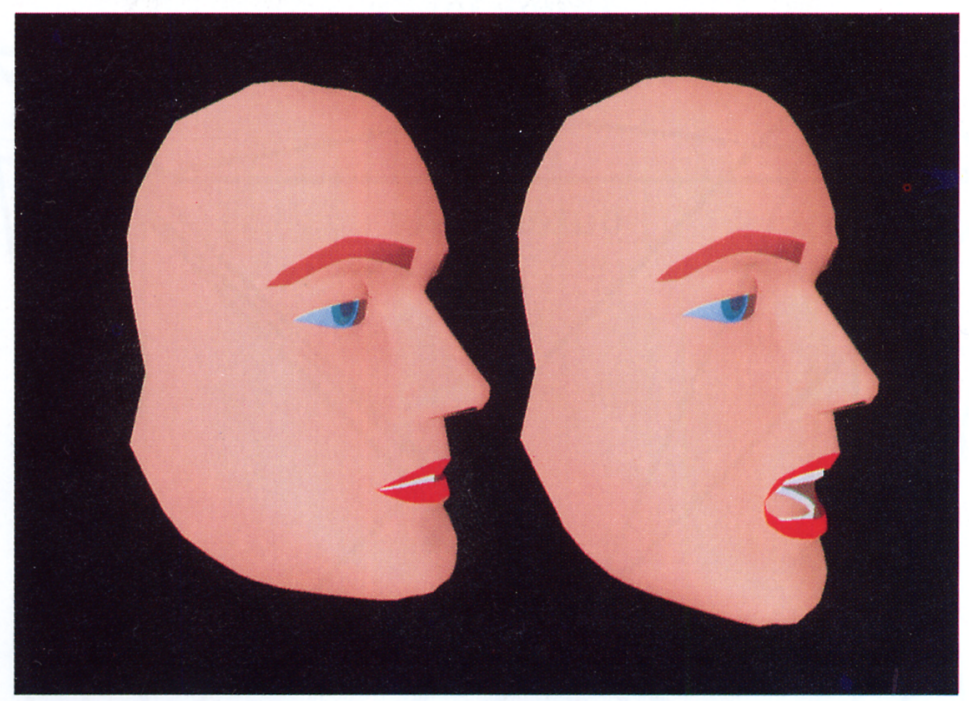

Fig. 3. Initial and final appearance of the face during jaw motion (open mouth).

simulated through time as follows. For each frame of an animation, the jaw joint moves and the inner layer moves as dictated by the jaw joint. The points in the outer layer (face skin) moves from their current positions to their new positions after a certain number of iterations of the differential equation solver, in other words, until the springs come to rest approximately.

The system of second order ordinary differential equations is integrated through time using different techniques such as explicit Euler, midpoint and fourth-order Runge-Kutta methods with adaptive time-stepping [9].

\section{SIMULATION OF EATING AND CHEWING ACTIONS}

To simulate eating, the mouth should be opened to a certain degree to take the food mass into mouth and should be closed after taking the food mass. I have simulated jaw motions for mouth opening and closing while the face skin and lip shapes remain realis ic $^{2}$. To make the shape of the lips appear

2 A movie file simulating the eating and chewing actions which can be played using movieplayer program on Silicon

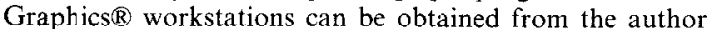
via ftp.

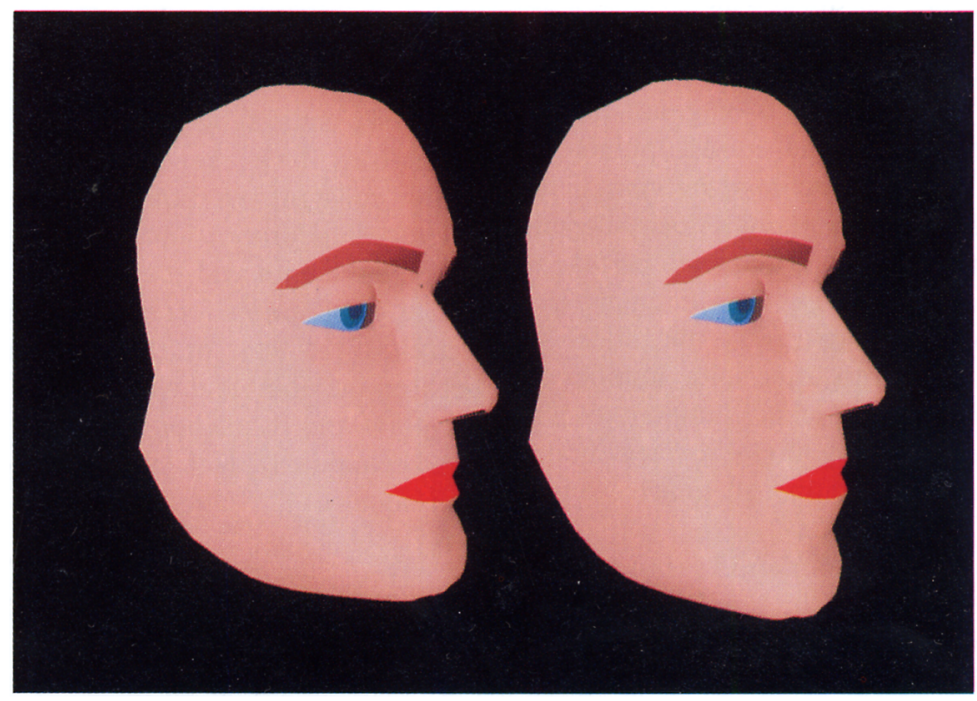

Fig. 4. Initial and final appenrance of the face during jaw motion (closed mouth). 


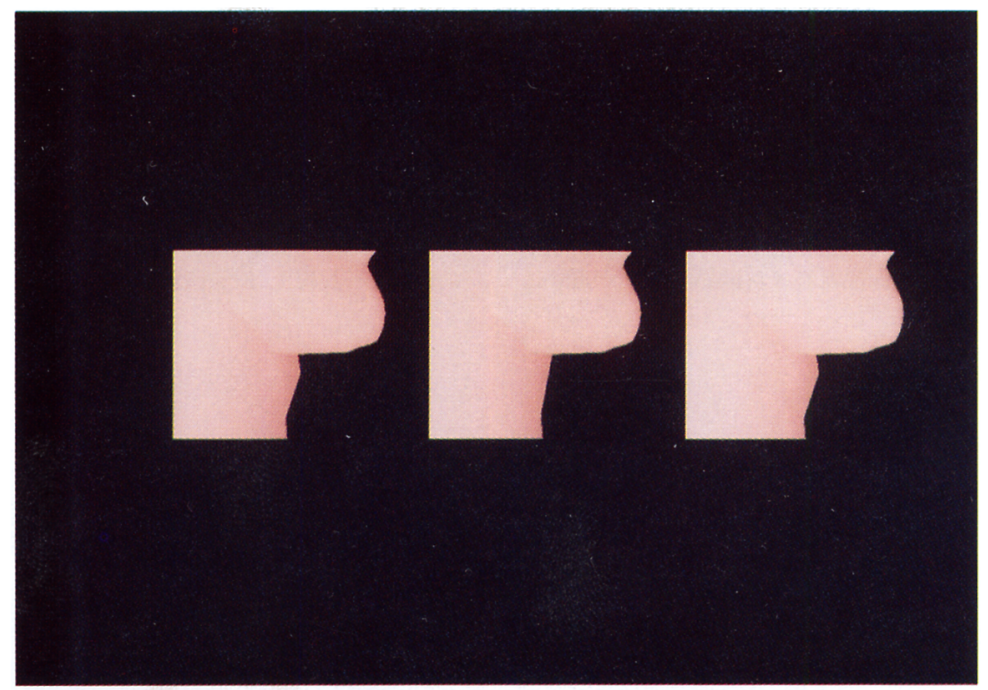

Fig. 5. Still frames of the adam's apple during the swallowing motion.

realistic, I put additional springs between the upper and lower lip points near the corners. Otherwise, the lower lip moves completely with the inner layer and separated from the upper lip on the corners during mouth opening. Figure 3 shows the initial and final appearance of the face during mouth opening.

The main problem in simulating eating and chewing is to do appropriate jaw motions while the mouth stays closed and the face skin remains connected and realistic. I simulate the chewing action as periodic opening and closing of the jaw joint up to a certain angle. To achieve realistic lip shapes, the lips are treated as rigid bodies. The lips are transformed as rigid bodies kinematically according to the movement of the jaw. In other words, I don "t change their positions during the solution of the differential equations. Figure 4 shows the initial and final appearance of the face during chewing action.

I also simulated another important feature of eating behavior, which is the swallowing motion. During the swallowing motion, when the food passes to the esophagus, the pomum adami (adam's apple) first moves upwards and then downwards. To achieve the swallowing motion, a throat is added to the face model and the coordinates of the points of the throat corresponding to the adam's apple are changed kinematically during the swallowing motion. Figure 5 shows still frames of the Adam's apple during the swallowing motion.

\section{CONCLUSIONS}

A movable jaw model is added to a synthetic face to simulate eating and chewing actions. Our face model is composed of upper and lower jaw bones, upper and lower teeth attached to the jaw bones, and a two-layer skin model. The inner layer of the face is attached to the jaw bones and moves kinematically as dictated by the jaw bones. The outer layer is attached to the inner layer with springs. Each outer layer node is also attached to its neighbors with springs. The main problem for chewing is to do appropriate jaw motions while the mouth stays closed and the skin remains connected and realistic.

The work in this paper could be extended to simulate other features of eating and chewing behavior. Some possible future extensions are:

- The arm motions could be simulated for insertion of food mass into mouth (feeding).

- Typical material properties of food stuff could be simulated. Different foods have different elasticity, stickiness and viscosity.

- Food mass reduction during chewing could be simulated.

Acknowledgements - I gratefully acknowledge Norman I. Badler and Dimitri N. Metaxas for reading the paper and providing valuable feedback. I thank Douglas DeCarlo for providing me the generic routines for solving second order ordinary differential equations. I used Stephen M. Platt's face geometry in my model. Thanks to Bülent Özgüç, Varol Akman, and Catherine Pelachaud for valuable discussions. Support of Advanced Research Projects Agency (ARPA DAMD17-94-J-4486) is gratefully acknowledged.

\section{REFERENCES}

1. Badler, N I., Phillips, C. B. and Webber, B. L. Simulating Humans: Computer Graphics Animation and Control. Oxford University Press, New York, 1993.

2. Baragar, F. A. and Osborn, W., A model relating patterns of human jaw movement to biomechanical constraints. J. Biomechanics, 1984, 17(10), 757-767.

3. Pelachaud, C., Badler, N. I. and Viaud M., Final Report to NSF of the Standards for Facial Animation Workshop. Technical Report IRCS-94-21, Computer and Information Science Department. University of Pennsylvania, October 1994. 
4. Parke, F. I., Parameterized models for facial animation. IEEE Computer Graphics and Applications, 1982, 2(5), 61-68.

5. Platt, S. M., A Structural Model of the Human Face. $\mathrm{PhD}$ Thesis, Computer and Information Science Department, University of Pennsylvania, 1985.

6. Terzopoulos, D. and Waters, K.. Physically-based facial modelling, analysis, and animation. The Journal of Visualization and Computer Animation, 1990, 1, 73-80.

7. Kunii. T. L., Karol Myszkowski, Okunev, O., Nishida,
H., Shinagawa, Y. and Ibusuki, M. Evaluation of Human Jaw Articulation. In Computer Animation'95, Geneva, Switzerland, April 1995, pp. 163-171.

8. Barzel, R., Physically-Based Modeling for Computer Graphics: A Structured Approach. Academic Press. Boston, MA, 1992.

9. Press, W. H., Flannery, B. P., Teukolsky, S. A. and Veterling, W. T., Numerical Recipes in C: The Art of Scientific Computing. Cambridge University Press. Cambridge, U.K., 1992. 